\section{Buchrezension zu: Kann Wissenschaft witzig?}

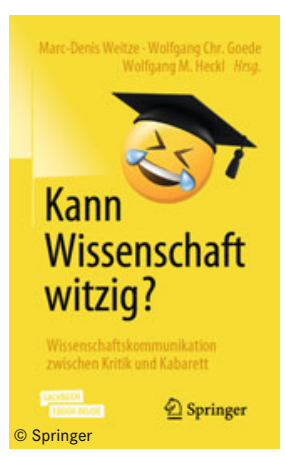

Kann Wissenschaft witzig? Wissenschaftskommunikation zwischen Kritik und Kabarett Marc-Denis Weitze, Wolfgang C. Goede, Wolfgang M. Hecke (Hrsg.)

266 S., Springer, 2021. SC, 20,00 €. ISBN: 9783662615812

Auch als E-Book erhältlich

DOI: 10.1007/s12268-021-1634-0 (C) Der Autor 2021

Witzigkeit kennt keine Grenzen, das wissen wir spätestens seit dem letzten Jahrtausend. Ob Witzigkeit auch vor der Wissenschaft nicht haltmachen kann oder darf und diese nachhaltig bereichert, muss jede:r Leser:in nach der Lektüre dieses Buchs von Marc-Dennis Weitze, Wolfgang Goede und Wolfgang Heckl für sich entscheiden. Ein Blick lohnt sich auf jeden Fall. Denn diese drei Herren, ihres Zeichens Wissenschaftler und erfahrene Lehrende bzw. Dozenten, haben dafür andere Herren eingeladen, ihre (wissenschaftliche) Komik sowie ihre Gedanken und Erfahrungen dazu in Beiträgen vorzustellen. Und so findet man neben sehr bekannten Namen wie Eckart von Hirschhausen und Jean Pütz auch eher unbekannte und jüngere Komödianten jeder Couleur. Allerdings mit Ausnahme einer Münchner Komikerin und einer spanischen Kabarettistin als Ko-Autorinnen keine Frauen; witzige Wissenschaft scheint auf den ersten Blick also entweder sehr männlich zu sein oder fast nur von Männern beherrscht zu werden.
Das Ziel der Herausgeber ist es, ein „Kochbuch“ mit Rezepten für den humorvollen Umgang mit Wissenschaft zu schreiben und zu zeigen, dass "die Wissenschaft" nicht immer bierernst, humorbefreit und hochgelehrt daherkommen muss. Das ist eine gute und wichtige Sache, denn Wissenschaft, die breitentauglich sein und rezipiert werden will, muss die Zuhörer:innen bzw. Leser:innen erreichen und eine Geschichte (ebenfalls bekannt als das Narrativ) daraus machen. Somit ist es im Ende ein Buch über Kommunikation aus Sicht der Sender, die sich versuchen in die Empfänger:innen hineinzuversetzen, Erfahrungswerte vorzustellen und die Leserschaft im besten Sinne auch zu unterhalten.

Ist das nun ein „fesselndes Feuerwerk" geworden? Humor ist subjektiv, und so mögen die einen Leser:innen die Beiträge und Beispiele witzig und hilfreich finden, die anderen sie eher für durchschnittlich unterhaltsam bis abwegig halten. Es sind gute Ideen und Ansätze für eine unterhaltsame, wirksame Wissenschaftskommunikation dabei. Sicherlich ist Komik als Kommunikationselement ebensowenig neu wie Wissenschaftshumor, doch die Verbindung zwischen den beiden ist relativ modern und frisch - und wichtig. Vermutlich hat nicht jede:r Lust, gleich einen Science Slam zu organisieren oder ein Lied mit gereimtem Text zu kreieren. Doch dem eigenen Vortrag, dem Artikel in einer Publikation oder der Präsentation im Seminar etwas mehr Würze mit Humor zu verleihen, sich Ideen aus dem Drehbuch zu holen und es mit Selbstironie zu versuchen, kann niemandem schaden.

Womit auch die Frage beantwortet ist, an wen sich die Herausgeber wenden: an alle, die Wissenschaft „machen“ und diese in die Welt bringen wollen, vom Beginn des Studiums bis zum professoralen Gipfel der akademi- schen Weihen. Probieren Sie es aus!

Jochen O. Ley,

Humboldt-Universität zu Berlin, jochen.ley@uv.hu-berlin.de

Diese Rezension erscheint Open Access. *

* Funding note: Open Access funding enabled and organized by Projekt DEAL. Open Access: Dieser Artikel wird unter der Creative Commons Namensnennung 4.0 International Lizenz veröffentlicht, welche die Nutzung, Vervielfältigung, Bearbeitung, Verbreitung und Wiedergabe in jeglichem Medium und Format erlaubt, sofern Sie den/die ursprünglichen Autor(en) und die Quelle ordnungsgemäß nennen, einen Link zur Creative Commons Lizenz beifügen und angeben, ob Änderungen vorgenommen wurden. Die in diesem Artikel enthaltenen Bilder und sonstiges Drittmaterial unterliegen ebenfalls der genannten Creative Commons Lizenz, sofern sich aus der Abbildungslegende nichts anderes ergibt. Sofern das betreffende Material nicht unter der genannten Creative Commons Lizenz steht und die betreffende Handlung nicht nach gesetzlichen Vorschriften erlaubt ist, ist für die oben aufgeführten Weiterverwendungen des Materials die Einwilligung des jeweiligen Rechteinhabers einzuholen. Weitere Details zur Lizenz entnehmen Sie bitte der Lizenzinformation auf http://creativecommons.org/licenses/ by $/ 4.0 /$ deed.de. 